Disclosure of Interest: None declared

DOI: 10.1136/annrheumdis-2017-eular.5143

\section{FRI0394 NEUROPATHIC PAIN IS ASSOCIATED WITH SKIN THICKNESS IN SYSTEMIC SCLEROSIS PATIENTS}

J. Sousa-Neves, M. Cerqueira, D. Santos-Faria, J. Leite Silva, C. Afonso, F. Teixeira. Rheumatology, Hospital Conde de Bertiandos, ULSAM, Ponte de Lima, Portugal

Background: Systemic Sclerosis (SSc) is a connective tissue disorder characterized by fibrosis of skin and internal organs, vasculopathy and systemic inflammation. To date, few studies assessed pain in SSc patients,(1) namely the neuropathic component. Data is also scarce on defining possible neuropathic pain (NP) predictors in these patients.

Objectives: To determine if patients with SSc have a higher prevalence of NP compared with a group of age and sex matched controls and study possible associations between NP and SSc clinical variables.

Methods: The study evaluated patients diagnosed with SSc fulfilling the SSc classification criteria of the American College of Rheumatology (ACR), older than 18 years old and followed up at our Rheumatology Unit. Forty-eight patients with mean age of $56.98 \pm 12.73$ years and mean disease duration of $9.77 \pm 6.12$ years were included. Comparison group comprised 45 age and sex matched controls. Patients and controls were consecutively evaluated at our Unit and NP in 4 questions questionnaire (DN4) was used for assessing the presence of NP. In SSc patients, skin involvement was also evaluated clinically by the modified Rodnan skin thickness score (mRSS) ranging from 0 to 51 . Hand mobility (HAMIS) and SSc Severity Scale (SScSS) were also calculated and relevant clinical variables of the disease were collected. Parametric and nonparametric tests were used for comparative analysis. Multivariate logistic regression was used to identify factors associated to NP. Statistical significance was defined as $\mathrm{P}<0.05$.

Results: In our study, prevalence of NP assessed by DN4 was significantly higher in SSc patients comparative to controls $(56.2 \%$ versus $13.3 \%, \mathrm{p}<0.001)$. In addition to age and sex, presence of diabetes $(p=0.541)$ was also similar in both groups. In SSc group, patients with and without NP showed some statistically significant differences (table 1).

Table 1. Comparative analysis between SSc patients with and without NP

\begin{tabular}{lccc}
\hline & $\begin{array}{c}\text { NP positive patients } \\
(\mathrm{n}=27)\end{array}$ & $\begin{array}{c}\text { No-NP patients } \\
(\mathrm{n}=21)\end{array}$ & P value \\
\hline Median age, years (IQR) & $61(57-69)$ & $52.38(42.5-63.5)$ & 0.032 \\
Mean disease duration (years) & $11.36 \pm 6.55$ & $7.73 \pm 4.93$ & 0.04 \\
Diffuse/limited cutaneous subtype (n) & $6 / 21$ & $0 / 21$ & 0.024 \\
Mean mRSS & $22.81 \pm 8.74$ & $7.57 \pm 3.64$ & $<0.001$ \\
Mean HAMIS & $10.44 \pm 6.16$ & $1.52 \pm 2.29$ & $<0.001$ \\
Mean SScSS & $10.00 \pm 4.32$ & $3.90 \pm 2.07$ & $<0.001$ \\
Digital ulcers n (\%) & $17(63.0)$ & $6(28.6)$ & 0.018 \\
Calcinosis n (\%) & $15(55.5)$ & $6(28.6)$ & 0.062 \\
\hline
\end{tabular}

Multivariate logistic regression revealed that only mean mRSS (odds ratio [OR] $=1.90,95 \%$ confidence interval $[\mathrm{Cl}] 1.01$ to $3.55, \mathrm{p}=0.045$ ) was independently associated with the presence of NP.

Conclusions: To the best of our knowledge, this is the first study evaluating the prevalence of NP in SSc patients in comparison with age and sex matched controls. NP was significantly more prevalent in patients with SSc. Skin thickness assessed by mRSS was independently associated with the presence of NP.

\section{References:}

[1] Schiei $O$ et al. Prevalence, severity, and clinical correlates of pain in patients with systemic sclerosis. Arthritis Care Res (Hoboken). 2010 Mar; 62(3):40917.

Disclosure of Interest: None declared

DOI: 10.1136/annrheumdis-2017-eular.5288

\section{FRI0395 INCIDENCE OF MYOSITIS-SPECIFIC AUTOANTIBODY (MSA) SPECIFICITIES IN SERA REFERRED TO NEW ZEALAND (NZ) MEDICAL LABORATORIES}

J. O'donnell ${ }^{1}$, P. Keating ${ }^{1}$, M. van Voorthuizen ${ }^{1}$, J. Liu ${ }^{1}$, I. Chua ${ }^{1}$, A. Soepnel ${ }^{2}$ E. Gumbley ${ }^{2}$, M. Spellerberg ${ }^{1} .{ }^{1}$ Immunology Section, Canterbury Health Labs, New Zealand, Christchurch; ${ }^{2}$ Waikato Hospital Laboratory, Waikato Hospital, Hamilton, New Zealand

Background: Idiopathic inflammatory myopathies can be classified by clinicopathological phenotype into four major groups: overlap myositis (OM), dermatomyositis (DM), immune mediated necrotising myositis (IMMNM) and inclusion body myositis (IBM). The different phenotypes associate with distinct MSA specificities although a variable percentage within each group are seronegative and the majority of IBM patients are seronegative ${ }^{1}$. No commercial assay for the IBM associated specificity, $\mathrm{cN} 1 \mathrm{~A}$, is available. In NZ testing for MSA is restricted to two laboratories: Waikato Hospital (WHL) and Canterbury Health Laboratories (CHL). Both laboratories use the commercial Euroimmun Euroline assay. In addition CHL has developed an ELISA for anti HMGCR autoantibodies and receives referrals from throughout NZ.
Objectives: To define the incidence and specificities of MSA in serum samples referred for testing in New Zealand.

Methods: For the period 3 November 2015 to 2 November 2016 each laboratory information system (LIS) was interrogated for requests for MSA. For the purposes of this report positive results were grouped into the specificities associated with each of the defined clinicopathological phenotypes.

DM: Mi2, Tif- $\gamma$, MDA 5 NXP2 SAE1

OM: Ku, PM-Scl-100, PL75, Jo1, PL-7, PL-12, EJ, OJ

IMMNM: HMGCR, SRP

Results: Of screened sera $(n=793, C H L=622, W H L=171) 11 \%$ were positive for MSA (88/793). Positive sera where distributed amongst three clinicopathological associated autoantibody specificities: DM 23\% OM 50\%,IMMNM 27\%. The most common serum specificities were HMGCR $(n=24)$ and Jo-1 $(n=13)$. No serum with specificities to SRP or OJ was detected. 22/24 sera positive for anti HMGCR had an elevated CPK concentration $(C P K>2500 u / l$ in $21 / 24$ and $C P K=544$ in $1 / 24$, reference range $=66-220$ ) .

Conclusions: In New Zealand $11 \%$ of sera referred for MSA testing were positive. As a group the most common specificities were those associated with $\mathrm{OM}$ while the most common antigen specificity was to HMGCR. Anti-HMGCR antibodies were almost invariably associated with major elevations in serum CPK.

References:

[1] Beveniste O, Stenzel W, Allenbach Y. Advances in serological diagnostics of inflammatory myopathies. Curr Opin Neurol 2016;29:662-673.

Disclosure of Interest: None declared

DOI: 10.1136/annrheumdis-2017-eular.2329

\section{FRI0396 LEVELS PENTRAXIN 3 AND C1Q IN SYSTEMIC SCLEROSIS: ASSOCIATION WITH PULMONARY ARTERIAL HYPERTENSION AND CLINICAL FINDINGS}

R. Cevik ${ }^{1}$, K. Nas ${ }^{2}$, T. Bozan ${ }^{1}$, A. Ekinci ${ }^{3}$, A. Kamanlı ${ }^{2}$, I. Tekeoglu ${ }^{2}$, M.O. Ayyildiz ${ }^{4}{ }^{1}$ Physical Medicine and Rehabilitation, Dicle University Hospital, Diyarbakir; ${ }^{2}$ Physical Medicine and Rehabilitation, Sakarya University, Faculty of Medicine, Sakarya; ${ }^{3}$ Department of Biochemistry; ${ }^{4}$ Internal Medicine, Dicle University Hospital, Diyarbakir, Turkey

Background: Systemic sclerosis (SSc) is an autoimmune rheumatic disease with unknown etiology that characterized fibrosis and vascular endotelial damage with obliteration of the microvasculature caused from exaggerated syntesis of collagen and extracellular matrix deposition in skin and internal organs. Pentraxin 3 (PTX3) accepted as a vascular inflammatory marker which secreted in early stage of endothelial dysfunction.

Objectives: In this study, we aimed to investigate plasma PTX3 and serum complement $\mathrm{C} 1 \mathrm{q}$ levels and their relations with cytokines and pulmonary arterial pressure in SSc.

Methods: Fifty nine patients diagnosed SSc and 28 subjects without inflammatory rheumatic disease recruited to the study. Plasma PTX3, and serum TNF- $\alpha$, IL-1 $\beta$, IL-4, IL-10, INF- $\gamma$, TGF- $\beta$ and complement C1q measured in both groups. Clinical findings and pulmonary arterial pressure of the SSc patients assessed.

Results: Ages of fifty nine with SSc (54 female and 5 male) and 28 control subjects ( 23 female and 5 male) were $49,47 \pm 12.74$ and $43,07 \pm 13,71$ respectively. PTX3, IL-1 $\beta$ and IL-4 levels found decreased in SSc patients. There were no differentiations between limited and diffuse cutaneous SSc subgroups in terms of PTX3, cytikines and C1q levels. Plasma PTX3 and serum IL-1 $\beta$ concentrations found decreased only limited cutaneous SSc subgroup comparing controls. According to the immunosupressive drugs use; PTX3 levels found decreased in using group and TNF- $\alpha$ ve IL-1 $\beta$ levels found decreased in not using group comparing controls. Significant positive association found between PTX3 and C1q and all cytokines except TGF- $\beta$.

Conclusions: PTX3 suggested to be used in the pathogenesis and assessment of disease treatment efficacy of SSc, because of found decreased in SSc patients especially in limited cutaneous and immunosupressive drugs using subgroups. Associations between C1q, and PTX3 and pulmonary artey pressure highlighted possible roles of both parameters in development of pulmonary hypertension. It is possible to develop preventive treatment strategies with better understanding of these associations. Advanced studies should be carried out for clarifying this condition.

Disclosure of Interest: None declared

DOI: 10.1136/annrheumdis-2017-eular.6755

\section{FRI0397 EVALUATION OF VITAMIN B12 DEFICIENCY AND ASSOCIATED FACTORS IN PATIENTS WITH SYSTEMIC SCLEROSIS}

D. Tas Kilic ${ }^{1}$, A. Sari ${ }^{2}$, B. Armagan ${ }^{2}$, A. Erden ${ }^{2}$, L. Kilic ${ }^{2}$, M. Kilinckaya ${ }^{3}$

T. Turhan ${ }^{3}$, A. Akdogan ${ }^{2}$, S. Karaahmetoglu ${ }^{1} .{ }^{1}$ Department of Internal Medicine, Numune Education and Research Hospital; ${ }^{2}$ Department of Internal Medicine Division of Rheumatology, Hacettepe University Faculty of Medicine; ${ }^{3}$ Department of Biochemistry, Numune Education and Research Hospital, Ankara, Turkey

Background: Vitamin B12 (Vit B12) deficiency is a common condition, which can be manifested with non-specific clinical features or with neurological and/or haematological abnormalities in severe cases. In systemic sclerosis (SSc) 